Cuadernos de Geografía • $107 \bullet 175-190 \bullet$ València 2021

RAMON J. BATALLA ${ }^{a}$

\title{
REFLEXIÓN SOBRE DINÁMICA MORFOSEDIMENTARIA. IMPLICACIONES PARA LA GESTIÓN FLUVIAL EN UN CONTEXTO DE CAMBIO GLOBAL
}

\begin{abstract}
RESUMEN
La conservación de la estructura y el funcionamiento de los cauces fluviales y, en su caso, su rehabilitación se debe sustentar en un profundo conocimiento de los procesos físicos que en ellos tienen lugar y el nexo con la cuenca de drenaje. En este sentido, es importante avanzar en el análisis detallado de los componentes morfodinámicos y sedimentarios claves que permitan definir, por ejemplo, umbrales de cambio irreversibles en medios fluviales poco o no alterados y programas de reconexión lateral y longitudinal en medios muy alterados, todo ello encaminado a la consecución del mejor estado ecológico y de conservación de los ecosistemas fluviales.

PAlabras Clave: dinámica morfosedimentaria; agua; sedimentos; rehabilitación funcional; continuidad fluvial.

\section{REFLECTION ON MORPHO-SEDIMENTARY DYNAMICS. IMPLICATIONS FOR RIVER MANAGEMENT IN A CONTEXT OF GLOBAL CHANGE}

\begin{abstract}
The conservation of the structure and functioning of river channels and, where appropriate, their rehabilitation, must be based on a sound knowledge of the physical processes that take place in them, and the intimate link with the drainage basin. Hence, it is important to move forward in the detailed

a Grup de Recerca de Dinàmica Fluvial (RIUS), Universitat de Lleida (UdL). 25198 Lleida. Institut Català de Recerca de l'Aigua (ICRA). 17241 Girona. Facultad de Ciencias Forestales y Recursos Naturales, Universidad Austral de Chile, Valdivia, Chile. ramon.batalla@udl.cat. http://orcid.org/0000-0001-8454-2314.
\end{abstract}

Fecha de recepción: 21-07-2021. Fecha de aceptación: 09-12-2021. 
analysis of the key morphodynamic and sedimentary components that allow defining, for example, irreversible change thresholds in fluvial environments less or no altered, and lateral and longitudinal reconnection programs in highly altered environments, all aimed to the achievement of the best possible ecological and conservation status of river ecosystems.

KeYwords: morphosedimentary dynamics; water; sediments; functional rehabilitation; river continuity.

\section{DiNÁMICA HIDROLÓGICA Y MORFOSEDIMENTARIA}

El conocimiento de la morfología y del transporte de sedimentos son el punto de partida para la conservación de la estructura y el funcionamiento de los ecosistemas fluviales. Los ríos se encuentran entre los sistemas más complejos y dinámicos de la naturaleza, y constituyen sistemas naturales caracterizados por transferencias más o menos frecuentes de agua y sedimentos a lo largo del continuo fluvial, que, a su vez, sustentan la vida. Mientras se mueven a través de cursos fluviales, el agua y los sedimentos conectan todos los compartimentos de la red de drenaje, desde las cabeceras de la cuenca a las zonas de deposición en las tierras bajas (Leopold et al., 1964; Richards, 1982), en un completo y complejo sistema de intercambio y transferencia de masa y energía, lo que configura la cuenca hidrográfica o fluvial. El hecho de no apreciar esta conexión fundamental subyace a muchos de los problemas ambientales en la gestión actual de los ríos.

La forma del río y los sedimentos crean y mantienen una variedad de hábitats en el río, que sustentan la vida de muchos organismos. Un hábitat fluvial se refiere al sustrato, a las propiedades del flujo de agua y a los residuos orgánicos, entre otros, que sustentan el ciclo vital de los organismos, es decir, animales y plantas que en ellos viven. Cada uno los hábitats (e. g., rápidos, pozas, barras) ofrece oportunidades a diferentes ensamblajes de organismos. Incluso dentro de un solo hábitat fluvial, hay muchos lugares donde los animales pueden vivir. La vegetación de ribera, es decir, las plantas que crecen en los márgenes inundables, es también un elemento importante en la arquitectura de los ríos y ejerce una gran influencia en su funcionamiento (Cowell \& Dyer, 2002; Cotton et al., 2006). Por lo tanto, las zonas ribereñas saludables son clave para que el hábitat sea completo. Los seres humanos modifican y alteran los ríos. Las alteraciones afectan al funcionamiento físico de los sistemas fluviales tanto por cambios de uso del suelo a escala de cuenca como por actividades dentro del cauce; entre estas actividades destacan la construcción de presas y la extracción de áridos de los cauces. Dichos impactos alteran el suministro de agua y sedimentos a la red de drenaje, así como la transferencia de masa y energía dentro del sistema. Los cambios en los usos del suelo (i. e., reforestación, deforestación, urbanización) afectan sobre todo a la escorrentía y a la producción y el suministro de sedimentos a largo plazo. Por su parte, las presas afectan al régimen de fluvial de agua y sedimentos a largo plazo y a largas distancias. La minería dentro del cauce, es decir, la extracción de sedimentos de ríos y llanuras de inundación adyacentes, actúa localmente agotando el cauce de sedimentos, y sus efectos pueden propagarse aguas arriba y abajo durante décadas (ver figura 1 para observar cambios compuestos en el patrón de drenaje consecuencia de la regulación por embalses y extracciones de áridos en un mismo tramo fluvial). 


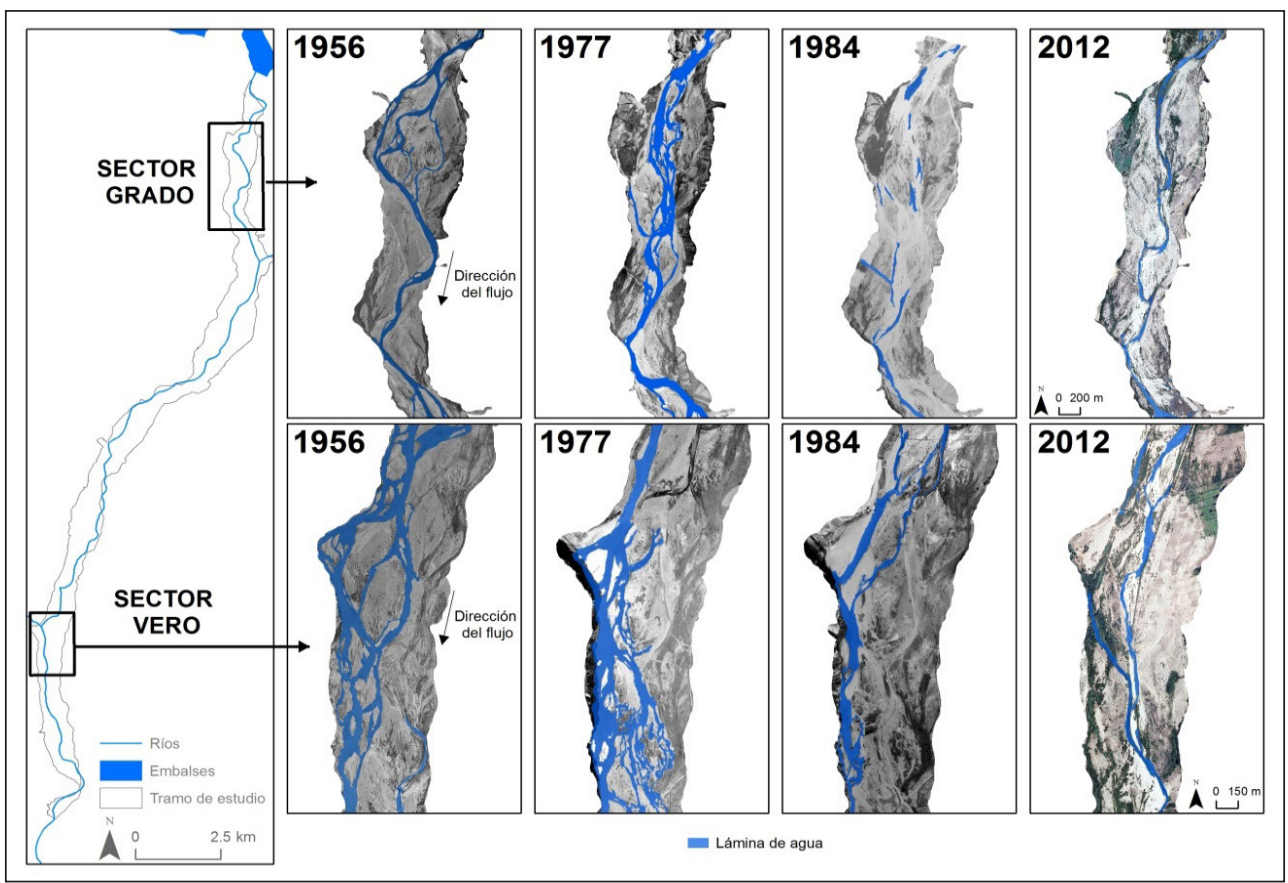

Figura 1. Ejemplo de cambio del patrón de drenaje y la conectividad lateral en un río afectado por presas y extracciones de áridos. Tramo medio del río Cinca (cuenca del Ebro).

La estructura de los cauces aluviales, es decir, su arquitectura básica está formada por sedimentos que experimentan ciclos de erosión, arrastre, transporte y deposición (Church, 2006). La mayoría de los ríos de la Tierra son aluviales, es decir, el agua corre entre mezclas sueltas de sedimentos que se han depositado previamente. Estos sedimentos forman la estructura básica de los ríos; dentro de ellos, el agua se mueve hacia arriba y en profundidad, y lateralmente en conexión directa con el agua subterránea en las llanuras de inundación. Estos sedimentos albergan una variedad de fauna y flora, y sostienen vegetación. La arquitectura de los cauces de los ríos está controlada por las interacciones entre el caudal líquido, el tamaño y la clasificación de los sedimentos, el suministro de nuevos materiales de la cuenca y su transporte río abajo (Leopold et al., 1964; Church, 2006). Estas interacciones controlan los cambios que los cauces de los ríos experimentan, así como su frecuencia y magnitud (Ligon et al., 1995; Kondolf, 1997). Es importante recordar, en este sentido, que las crecidas son perturbaciones físicas fundamentales para los organismos que habitan los ríos, más allá de los potenciales daños que puedan causar a bienes y personas.

El objetivo de este artículo es ofrecer una breve y general reflexión sobre la importancia de analizar correctamente la interacción entre las fuerzas del flujo de agua y los sedimentos que dan forma a los ríos, y su relación con el funcionamiento del ecosistema. El análisis de los procesos físicos proporciona un marco integral para las ciencias fluviales, que permite ver el agua, los sedimentos y las características 
físicas resultantes como elementos fundamentales para comprender las medidas de conservación y restauración en el sistema e informar de estas. La conservación y, en su caso, la restauración comienza con la comprensión de los procesos físicos y la dinámica de los ríos. La gestión de ríos que descuida centrarse en los balances de masa (agua y sedimentos) y energía, como los factores que determinan la estructura y el funcionamiento de los ríos, está destinada al fracaso.

\section{Medición, EXPERIMENTACión Y MODELIZACióN}

De manera natural, las cuencas y los ríos están en proceso permanente de cambio y los impactos alteran además la naturaleza de ese cambio y dificultan la capacidad de los investigadores para analizar y predecir las trayectorias de cambio (respuesta, adaptación). La toma y el registro de datos es una parte fundamental del método científico, ya sea en su versión empírico-inductiva como en versión deductiva. En el primer caso, los datos ayudan a analizar relaciones causa efecto; por ejemplo, en un río cabe estudiar cómo el inicio del movimiento del material de fondo o el incremento del sedimento en suspensión activan la deriva de los macroinvertebrados. En el segundo caso, los datos permiten validar hipótesis ya testadas en otros medios fluviales anteriormente, por ejemplo, sobre la bondad de generar caudales ambientales para aumentar la conectividad lateral en un tramo de río que ha experimentado procesos crónicos de incisión. La toma de datos debe ser idealmente planificada a largo plazo para que los datos incluyan la máxima variabilidad posible, especialmente en cuencas con regímenes hidrológicos altamente variables como los de las regiones mediterráneas. Los datos hidrológicos que facilitan los organismos de cuenca (confederaciones, agencias) son fuentes de información muy relevantes, aunque el diseño y las mediciones no obedecen generalmente a criterios científicos sino de cuantificación y control de los recursos.

Una de las características más sobresalientes de la geomorfología moderna fue la adopción de los métodos cuantitativos, que han dado posteriormente lugar a una geomorfología de base cuantitativa y matemática (Scheidegger, 1961; Carson \& Kirkby, 1972). Tal y como reseñan Sala y Batalla (1996), uno de los aspectos más sobresalientes ha sido la incorporación amplia y progresiva a la investigación de las mediciones y experimentaciones en el campo y en el laboratorio. Si bien las instalaciones pioneras, y que todavía siguen en curso, se remontan a los años cincuenta del pasado siglo, no fue hasta los años setenta cuando su utilización se generalizó, por tratarse de una metodología que se considera indispensable para el estudio de los procesos geomorfológicos (Slaymaker, 1980; Sala \& Gallart, 1988). Los llamados experimentos de campo sirven para aportar datos sobre la magnitud y frecuencia de acción de los procesos y contribuyen a establecer sus variaciones espaciales y temporales. Las mediciones de la acción de un proceso bajo el control artificial de alguna de las variables se pueden considerar un experimento, puesto que se realiza en condiciones controladas, similares a las que se pueden obtener en un laboratorio. Los experimentos de campo son todavía poco abundantes entre los geomorfólogos, si bien son comunes entre hidrólogos, agrónomos e ingenieros, aunque la geomorfología ha avanzado también en esa dirección. Aun así, trabajos como los de Calvo (1988) mediante el uso de simuladores artificiales de lluvia que permitían cuantificar la erosión o los de Gibbins, Vericat y Batalla (Gibbins et al., 2007; Vericat et al., 
2008) son ejemplos que indican que la geomorfología está también avanzado en el campo de la experimentación, y en el caso particular de estos, los resultados contribuyeron a reforzar líneas específicas de la geomorfología fluvial en colaboración con otras disciplinas, en este último caso, la ecología.

El estudio de los procesos geomorfológicos, en su inicio cualitativo y posteriormente basado en la cuantificación y la experimentación, desembocó en la utilización de modelos matemáticos, diseñados para determinar o deducir la relación entre proceso y forma, entre causa y efecto, es decir, entre actividad geomorfológica y respuesta del cauce a su impacto. La modelización de procesos hidrosedimentarios mediante herramientas matemáticas con el apoyo de los sistemas de información geográfica, junto con el uso de técnicas geomáticas, que permiten obtener datos de muy alta resolución (Brasington et al., 2012), constituyen herramientas potentes para el análisis de procesos en ríos y la determinación de sus respuestas tanto a escenarios de cambio global (clima) como a aquellos que se dan aguas arriba (embalses, usos del suelo).

\section{INTERACCIONES BIOFÍSICAS Y HÁBITAT FLUVIAL}

Las características físicas controlan las condiciones del hábitat y son esenciales para mantener la diversidad ecológica de un sistema fluvial. La diversidad ecológica de los sistemas fluviales está directamente relacionada con la heterogeneidad de condiciones físicas del hábitat, incluida la hidráulica del flujo y el sustrato. Sin embargo, existen importantes consideraciones de escala relacionadas con el tamaño del organismo. Para los invertebrados, la escala relevante de heterogeneidad física es la del parche (es decir, de centímetros a metros), mientras que los peces, que son más grandes y móviles, dependen de heterogeneidad a escala de alcance, de cientos de metros a kilómetros (Poff, 1997). Los sedimentos presentan tamaños (granulometrías) y formas diferentes, y son la base de la compleja arquitectura de los cursos de agua. La heterogeneidad del sustrato y la complejidad topográfica son requisitos para la diversidad ecológica, que a su vez está vinculada a la salud (funcionamiento) de los ríos. La vida en los ríos requiere complejidad, estrés y, consecuentemente, perturbaciones y cambio. Los sedimentos de lechos fluviales relativamente inmóviles (es decir, cantos rodados, bloques) son hábitats importantes para invertebrados. Estas partículas ofrecen un hábitat más diverso para la colonización y mejores recursos alimentarios, i. e., retienen más materia orgánica y el biofilm se desarrolla mejor que en ambientes menos estables. Por ejemplo, los cauces con arena y grava son más móviles, y pequeños aumentos en el caudal mueven partículas y provocan la deriva de los animales bentónicos. Por lo tanto, la diversidad de hábitats depende de la disponibilidad de áreas inestables en el cauce, así como de refugios y aguas tranquilas. En ríos con alto contenido de sedimentos finos, la sedimentación entre las gravas bloquea el transporte de oxígeno y puede provocar la muerte de huevos de peces (Schälchli, 1992). Además, la obstrucción de los lechos por sedimentos finos también reduce la diversidad de invertebrados (Gibbins et al., 2007). En cualquier caso, los lechos de los ríos experimentan perturbaciones (crecidas) de manera episódica, cuya frecuencia y magnitud dependen del clima y de las características de la cuenca. La hidrología y dinámica sedimentaria interactúan para controlar la diversidad y funcionalidad del hábitat, en toda clase de medios fluviales, desde los ríos temporales a los perennes. 


\section{EFectos DEL CAMBIO GLOBAL SOBRE LOS PROCESOS HIDROSEDIMENTARIOS}

La cantidad de sedimentos que un río transporta y su tamaño están relacionados con la energía del flujo de agua (caudal), expresada por una combinación entre el caudal y la pendiente del río. Esta relación se expresa comúnmente a través de la balanza de Lane (Lane, 1955) y muestra que un cambio en cualquiera de las variables provoca un cambio en las demás hasta que se restablezca el equilibrio. Cuando un cauce está en equilibrio (dinámico) o cuasiequilibrio, el sedimento se transporta sin erosión ni sedimentación significativas en el lecho, i. e., sin cambios notables en la topografía, la cota de fondo y la pendiente de equilibrio. Cabe señalar, sin contradecir esta definición de equilibrio, que un cauce es libre de migrar lateralmente erosionando una de sus orillas y acumulando sedimentos en la opuesta a una tasa similar. Cuando el suministro de agua o sedimentos cambia, la geometría del cauce y la granulometría del lecho se ajustan. Estos cambios pueden ser el resultado de causas diversas, desde cambios en las tasas de erosión en la cuenca hasta cambios en el clima. Los cambios en la geometría del cauce también ocurren en relación con las oscilaciones del caudal durante el año (ciclos estacionales), pero estos cambios suelen ser menores.

\section{Cambio climático y cambio en los usos del suelo}

Los efectos del cambio climático y la intervención humana en el territorio sobre los procesos continentales (ciclos del agua y de sedimentos) son notablemente intensos en ciertas regiones, y esto es particularmente cierto en el conjunto de las regiones mediterráneas (Morán-Tejeda et al., 2010). Estas regiones experimentan escasez estructural de agua por las características intrínsecas de su clima, y también por el impacto humano creciente y duradero sobre los recursos hídricos (Conacher \& Sala, 1998; Sabater \& Barceló, 2012). Los recursos hídricos están controlados por la gran variabilidad interanual y estacional en el régimen de lluvias, así como por el clima cambiante, por las altas temperaturas y una menor precipitación (Milly et al., 2005). En zonas de montaña, estos impactos vienen acompañados de cambios socioeconómicos como el abandono de las actividades agrícolas, en particular durante la segunda mitad del siglo xx. Por ejemplo, en el Pirineo, se ha producido un abandono generalizado de la agricultura, que, junto con una menor presión humana sobre los bosques y pastos, ha favorecido la expansión del área boscosa (Grove \& Rackham, 2001). De hecho, los efectos de la expansión forestal han sido tales que se considera que es uno de los principales factores que impulsan la reducción de la escorrentía en las cuencas del península (Gallart \& Llorens, 2004; Morán-Tejeda et al., 2010), así como también de la producción y transferencia de sedimentos en las grandes cuencas (Vericat \& Batalla, 2006). Aunque los impactos hidrológicos del cambio del clima y de los usos del suelo parecen obvios, los estudios que evalúan tendencias de las series climáticas e hidrológicas durante el siglo xx han llegado a conclusiones divergentes. Mientras que algunos han informado de una intensificación de la escasez de agua en la región como consecuencia de las oscilaciones climáticas y el aumento de la demanda de agua (Mimikou et al., 2000), otros no han encontrado tendencias generalizadas (De Castro et al., 2005). En España, un estudio del CEDEX (2011) predice una reducción generalizada de 
las precipitaciones y la disponibilidad de agua, así como un aumento de la temperatura, de la evapotranspiración y una disminución en la recarga de agua subterránea y la escorrentía superficial. Según esto, la reducción en los recursos hídricos puede alcanzar el 5,9 y 17 \% para los periodos 2011-2040, 2041-2070 y 2071-2100, respectivamente. Definir la relación entre la escorrentía de la cuenca, los factores climáticos y del uso del suelo es crucial para desarrollar una gestión sostenible de las cuencas hidrográficas y definir e implementar la política del agua. Los cambios hidrológicos modifican a su vez el balance de sedimentos de la cuenca, con una reducción de la aportación sedimentaria media en los ríos debido a la disminución de la producción y transferencia de sedimentos desde la cuenca. La menor erosión resultante puede tener, no obstante, un impacto mayor en el transporte si los eventos extremos aumentan de magnitud y frecuencia (Buendía et al., 2016).

Los episodios hidroclimáticos de muy alta magnitud suponen una reforma completa de la estructura física (morfología) y un reinicio de su funcionamiento como ecosistema (Batalla et al., 2020). Cuando los caudales asociados a precipitaciones extremas alcanzan magnitudes muy importantes, provocan cambios profundos en la morfología de los cauces, inundando llanuras de inundación, reconectando brazos abandonados, provocando fenómenos de incisión generalizados y ensanchando ampliamente los cauces. Todo ello contribuye a reactivar los sistemas fluviales y añade un estadio más en el proceso de continuo metamorfismo que estos dinámicos sistemas experimentan. Los fenómenos hidroclimáticos extremos encuentran actualmente ríos constreñidos por la actividad antrópica continuada durante décadas, con los balances de sedimentos negativos y en una situación de fuerte desconexión hídrica y sedimentaria con la cuenca (cambios de usos del suelo y profundas alteraciones de la red de drenaje). Aun así, en ocasiones son suficientes solo unas horas de lluvia intensa, no necesariamente extrema, y la consiguiente rápida generación de caudales importante para aportar la energía efectiva capaz de superar muchos de los umbrales físicos que se les había ido imponiendo y cambiar completamente la morfología de los cauces y valles afectados (Batalla et al., 2020). La reacción de los sistemas fluviales altamente modificados ante un cambio en la magnitud y frecuencia de eventos hidroclimáticos extremos es todavía desconocida y solo la investigación a largo plazo de dichos procesos, sobre todo del comportamiento de la carga sólida y de los ajustes morfológicos de los cauces a través de herramientas de monitorización de alta resolución y modelos adecuadamente calibrados, permitirán determinar la magnitud del cambio y la respuesta de los ecosistemas.

\section{Regulación fluvial y producción de energía}

Los embalses atrapan una parte importante de la carga sedimentaria de los ríos, sobre todo las fracciones gruesas que se mueven en contacto con el lecho como carga de fondo. El aterramiento causa una progresiva reducción de la capacidad de embalse (figura 2) y puede generar problemas para la gestión del agua, especialmente cerca de las tomas de agua. La calidad del agua almacenada también se puede degradar (eutrofización). A su vez, la reducción del transporte de sedimentos altera el balance aguas abajo y genera cambios en la morfología y ecología fluviales. La naturaleza, extensión y propagación de estos dependen del tamaño de la presa, del grado de regulación, de las características fisiográficas y 
la operatividad del embalse, y del grado de afectación de los regímenes de caudales y sedimentos. Las presas liberan generalmente "agua hambrienta" (hungry water) (Kondolf, 1997), agua clara carente de sedimento y sin reposición de aluviones movilizados, que puede excavar el fondo y transportar material río abajo sin reemplazo desde aguas arriba, modificando la pendiente de energía y el balance de masas en el cauce, lo que genera incisión a corto plazo y erosión lateral a medida que el lecho se va acorazando (Vericat y Batalla, 2006). Se trata de procesos en muchos casos concomitantes y con feedback importantes, adaptándose este modelo evolutivo general a las características físicas particulares de cada tramo de río. Los efectos sobre la ecología fluvial son también conocidos, como la pérdida de zonas de freza para los peces, igual que los efectos sobre la vegetación de ribera, como la colonización y estabilización del corredor fluvial por especies endógenas y exógenas. Además, las presas reducen generalmente la magnitud y frecuencia de las crecidas, con lo que disminuye de esta manera la capacidad de transporte de los caudales (figura 3; Batalla et al., 2004) y con ello la carga sedimentaria a zonas litorales (Inman, 1976; Williams \& Wolman, 1984). La sedimentación pierde el equilibrio con la erosión costera, y formas como los deltas pueden retroceder (e. g., la erosión del delta del Nilo se cuantifica en $150 \mathrm{~m}$ por año, $1.000 \mathrm{~km}$ aguas abajo de la presa de Aswan; Brownlie \& Taylor, 1981). En España, existen alrededor de 1.200 grandes presas (capacidad total ca. $60 \mathrm{~km}^{3}$ ) y después de China y Estados Unidos es el tercer país con mayor número de ellas. Una parte muy importante tiene una antigüedad de medio siglo o más y, por lo tanto, los problemas ambientales relacionados con los sedimentos, singularmente su pérdida de capacidad, son de gran interés. Aunque no sea su uso principal, muchas presas almacenan agua para producir electricidad, producción que se genera específicamente o durante la suelta de agua para otros usos (canales de regadío, suministro urbano). La energía hidroeléctrica es el mayor contribuyente individual a la producción de energía renovable en el mundo $(76 \%)^{1}$ y proporciona el $16 \%$ de la electricidad global ${ }^{2}$. El consenso internacional para reducir las emisiones de gases de efecto invernadero garantiza un papel importante para la hidroelectricidad a medio plazo. Pero a pesar de ser clasificada como una forma verde de producción de energía, esta tecnología no está libre de impactos ambientales (Batalla et al., 2021). Las presas y las infraestructuras asociadas para la producción imponen presiones específicas en los ríos. No todas las presas tienen el mismo régimen operativo. Algunas producen lo que se denominan regímenes de "hidropuntas", que son aumentos súbitos del caudal en respuesta a la turbinación impuesta por la demanda de electricidad. Aunque muy poco estudiados, los efectos de las hidropuntas sobre el transporte de sedimentos y la movilidad de las partículas de lecho empiezan a ser conocidos (Vericat et al., 2020) y son una fuente importante de información para la mejora de la gestión fluvial en tramos afectados por estas instalaciones (1.312 en España, 1/3 de ellas en la cuenca del Ebro, con una potencia total instalada de ca. $18.000 .000 \mathrm{~kW}^{3}$ ).

$1<$ https://www.worldenergy.org/news-views/entry/new-world-energy-council-report-hydropoweras-big-surgeset-to-double-by-2050>.

$2<$ https://www.nationalgeographic.com/environment/global-warming/hydropower/>.

$3<$ http://hispagua.cedex.es/datos/energia> 


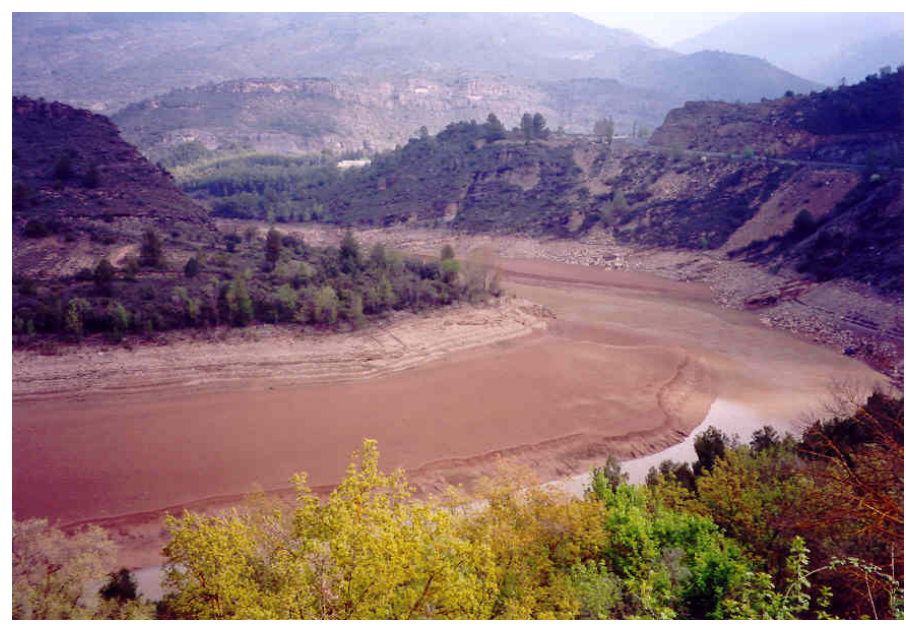

Figura 2. Sedimentos en el embalse de Camarasa, Noguera Pallaresa, Pirineo Central. Presa construida en 1920 (Foto: Ramon J. Batalla)

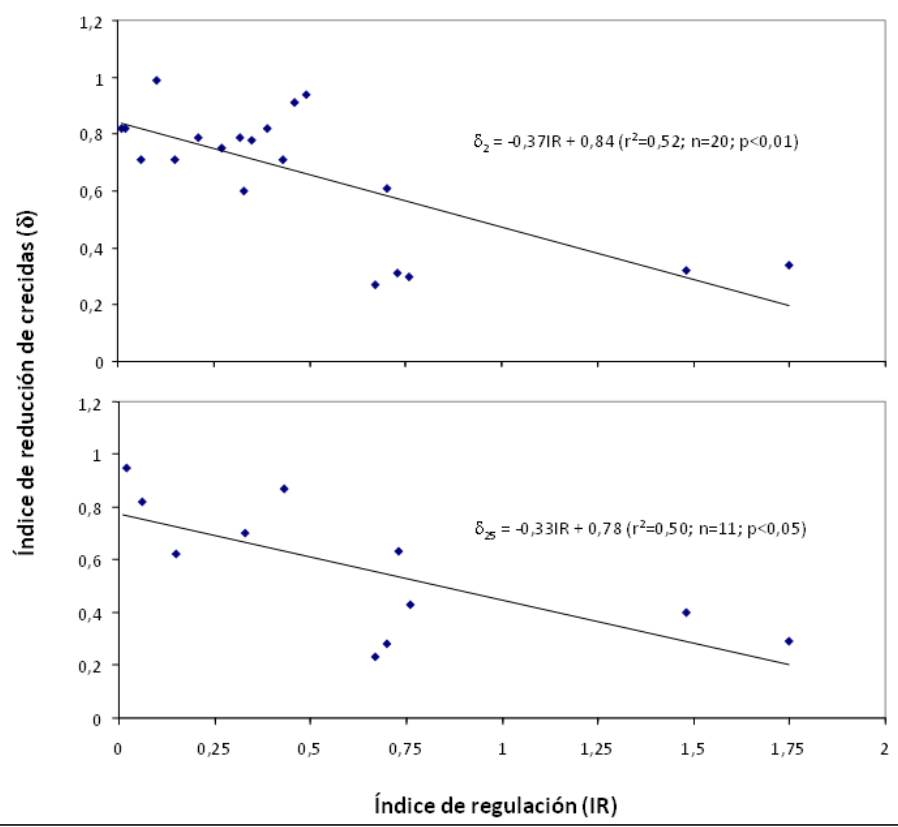

Figura 3. Reducción de crecidas en ríos de la cuenca del Ebro en relación con el grado de regulación. La reducción de crecidas ( $d$ ) se expresa como el cociente entre la magnitud de las crecidas antes y después de la construcción de la presa para un periodo de retorno de 2 años (arriba) y 25 años (abajo). Fuente: Batalla et al., 2004. 


\section{Extracción de áridos}

Como se ha indicado anteriormente, la morfología del cauce y su balance sedimentario (y, por lo tanto, las condiciones del hábitat y su diversidad ecológica) se reajustan cuando se modifica la disponibilidad de agua y sedimentos. La extracción de sedimentos tiene efectos directos (y localizados) en dichas condiciones al reducir el volumen de sedimentos almacenado (y con ello su disponibilidad) y, sobre todo, alterar la morfología en un determinado tramo del río. Aunque estos son los efectos más obvios y directos, las alteraciones físicas y ecológicas son múltiples y se interrelacionan de forma compleja. Erskine (1997) informó que tanto los impactos físicos como los ecológicos tienen una firma a corto y largo plazo. Los primeros son aquellos relacionados directamente con la actividad minera directa: por ejemplo, la creación de nubes de turbidez o barreras de agua para la migración de peces (Béjar et al., 2018). Los principales efectos morfológicos son la incisión en el cauce (Marston et al., 2003; Rovira et al., 2005), con importantes consecuencias sobre la estabilidad de puentes y otras infraestructuras, la inestabilidad de los cauces (Wyzga et al., 2009) y el acorazamiento del lecho (Erskine \& Green, 2000). En términos ecológicos, los impactos actúan homogeneizando las condiciones del hábitat (Wyzga et al., 2009); tanto la teoría ecológica como la evidencia empírica indican que esta homogeneización conduce a la reducción de la diversidad y a cambios en la composición de especies (e. g., macroinvertebrados, biofilm y peces; Brown et al., 1998). La investigación sobre los efectos de la extracción de gravas se ha concentrado en Estados Unidos (principalmente en California y Washington; e. g. Kondolf et al., 2002), en países europeos como Italia (Rinaldi \& Simon, 1998) y Francia (Piégay \& Peiry, 1997) y en Australia y Nueva Zelanda (Erskine, 1997). En España, los ríos han constituido desde los años sesenta la principal fuente de áridos para la construcción, lo que ha dado como resultado un agotamiento de la reserva sedimentaria aluvial de la mayoría de los ríos en las últimas décadas. Solo como ejemplo, la minería en el río Tordera, en el litoral de Cataluña, supuso una extracción de 5 millones de $\mathrm{m}^{3}$ entre 1956-1987, volumen excesivo para un río cuya carga sedimentaria media es $40.000 \mathrm{~m}^{3} / \mathrm{año}$ (Rovira et al., 2005). Este hecho no solo dejó en desequilibrio el balance de sedimentos fluvial durante siglos, y entre este y la costa (delta, playas), sino que alteró completamente el funcionamiento hidrogeológico y del ecosistema en su conjunto de una manera muy acusada. Actualmente, no se autorizan extracciones directas en el cauce con fines comerciales, excepto en casos relacionados con obras de protección contra inundaciones.

\section{El PARADIGMA DE LA REHABILITACIÓN FUNCIONAL}

En las últimas dos décadas, la restauración de ríos se ha convertido en un campo de investigación que plantea una serie de preguntas complejas relacionadas no solo con la ciencia sino también con la sociedad. Dufour y Piégay (2009) se plantearon el alcance de preguntas como estas: ¿Por qué deberíamos restaurar los ecosistemas? ¿La restauración siempre es beneficiosa? ¿Cuándo es beneficiosa? ¿Cuáles deberían ser los estados de referencia? ¿Qué es el éxito y cuándo se puede evaluar? Los autores plantearon una discusión sobre la dicotomía entre referencia y objetivo, estado ecológico versus objetivo 
de restauración, e integridad del ecosistema versus restauración impulsada para aumentar los servicios ecosistémicos; y argumentaron que, aunque el deseo de recrear las condiciones del pasado es tentador, la ciencia ha demostrado que los sistemas fluviales siguen trayectorias complejas que frecuentemente hacen imposible volver a un estado anterior. Por lo tanto, los objetivos de restauración se han ido alejando paulatinamente de la definición explícita de un estado de referencia debido a la dificultad de alcanzar dicho estado. La estrategia basada en referencias debe ser reemplazada progresivamente por una estrategia basada en objetivos que refleje las limitaciones prácticas del desarrollo de ecosistemas sostenibles y la importancia de tener en cuenta sus servicios. Después de décadas durante las cuales los procesos naturales fueron el foco de la restauración, se empezó a comprobar que dichos procesos no eran igualmente valiosos en todas partes y que a menudo eran inalcanzables, como mínimo de manera genérica, y que la complejidad regional y local debe entenderse mejor para ajustar las acciones de restauración (Dufour \& Piégay, 2009). Sobre esta base se han desarrollado líneas de trabajo que han ido consolidando la idea de la rehabilitación (funcional), hasta convertirla en paradigma, en contraposición al concepto genérico de restauración fluvial. En este sentido, Yarnell et al. (2015) señalaron la importancia de los regímenes de caudales ambientales sobre la ecología, así como el reconocimiento de que los procesos hidrogeomórficos son inherentes al funcionamiento ecológico de los ríos. Estos autores proponen un enfoque de caudales funcionales para la gestión de ríos impactados. El enfoque se centra en retener componentes específicos del hidrograma basados en procesos, o flujos de agua funcionales, en lugar de intentar imitar el proceso natural completo del régimen de caudales. Los componentes funcionales incluyen los caudales de iniciación de la estación húmeda, los de magnitud máxima, los de recesión, los caudales bajos de la estación seca y la variabilidad interanual. Para maximizar la funcionalidad de estos flujos, se debe mejorar la conectividad entre zonas del río morfológicamente diversas, tanto en el espacio como en el tiempo, y debe tenerse en cuenta el régimen de transporte de sedimentos, incluso si es necesaria su generación artificial. Batalla y Vericat (2009) desarrollaron e implementaron esta misma idea en el diseño de crecidas de mantenimiento en el tramo bajo del Ebro, basados en trabajos previos de Kondolf y Wilcock (1996), con el objetivo de revitalizar el transporte de sedimentos en un río altamente acorazado y con ello mejorar el funcionamiento general del ecosistema a través de la remoción de macrófitos que actúan como un factor de máxima estabilidad en el cauce.

\section{Consideraciones finales E INTERrogantes}

La principal conclusión que se puede extraer del estado de la cuestión es que la magnitud del cambio y del número de estresores que actúan simultáneamente sobre los sistemas fluviales probablemente no tiene un punto de referencia en el pasado, y que por ello es necesario doblar los esfuerzos de investigación, planteando, si cabe, aún más proyectos interdisciplinares para el estudio de estos sistemas altamente complejos. El marco legislativo actual (e. g., Directiva Marco del Agua 2000/60/CE del Parlamento Europeo y del Consejo, del 23 de octubre de 2000) permite avanzar en esta dirección y, en este sentido, la posición de la geomorfología fluvial puede jugar un papel importante, que permita aglutinar avances en la toma de datos y el tratamiento de estos, en el desarrollo y la implementación 
de metodologías de vigilancia y registro de procesos biofísicos y sus alteraciones, y en el desarrollo y validación de modelos para mejorar las previsiones a medio y largo plazo. En este contexto podrían plantearse diversas cuestiones para intentar catalizar el conocimiento adquirido durante décadas, avanzar en objetivos de rehabilitación funcional y dirigir posibles objetivos de trabajo en el futuro:

- ¿Cuáles son los componentes hidromorfológicos (e. g., régimen de caudales líquidos y sólidos, (in)estabilidad morfológica del cauce, magnitud y frecuencia de las perturbaciones naturales e inducidas), cuyo estudio debe ser clave para la consecución del mejor estado ecológico y de conservación en los ecosistemas fluviales?

- ¿Qué beneficios para el funcionamiento de los ecosistemas fluviales aportaría a medio y largo su progresiva reconexión lateral y longitudinal, así como la restauración de la continuidad sedimentaria, en tramos fluviales altamente modificados?

- ¿Cuáles son los umbrales de cambio irreversibles para caudales y sedimentos en medios fluviales menos alterados?

Para responder a estas cuestiones, la investigación fluvial deberá avanzar en diversos frentes intentando aglutinar el conocimiento de ciencias complementarias o auxiliares, que permita profundizar en 1) el análisis del transporte de sedimento y procesos fluviales asociados (i. e., morfodinámica, hidráulica de los flujos, estructura del cauce) en cursos de régimen permanente, estacional y efímero, a través de técnicas avanzadas de medición y modelización ; 2) el estudio mediante trabajo de campo, análisis y modelización de procesos hidrosedimentarios en ríos regulados, especialmente en aquellos que drenan grandes cuencas, y en los que el estado de sus ecosistemas constituye un tema de especial interés para la gestión ambiental, por ejemplo, para el diseño de crecidas de mantenimiento; y finalmente, 3 ) el conocimiento de las interacciones entre la hidráulica del flujo (e. g., energía y velocidad de la corriente), el transporte de sedimento y la estructura del cauce (balance de masas y hábitat físico), y las comunidades bentónicas de macroinvertebrados y peces (estado ecológico), hecho que permitiría entender mejor las relaciones biofísicas (ecogeomorfológicas) de los sistemas fluviales.

\section{Agradecimientos}

Este trabajo se beneficia de los resultados de investigación obtenidos en el marco de los proyectos CGL2006-11679-C02-01/HID y CGL2009-09770 (subprograma BTE) financiados por el Ministerio de Educación y Ciencia. El Grupo de Investigación de Dinámica Fluvial-RIUS agradece el apoyo de la Generalitat de Catalunya a través del grupo de investigación (2017 SGR 0459) y el programa CERCA. El autor reconoce y agradece a Damià Vericat y Álvaro Tena y al conjunto del grupo de investigación RIUS su tarea como investigadores, cuyos resultados, entre otros, han permitido elaborar este artículo de revisión y reflexión. Álvaro Tena elaboró la figura 1 de este trabajo. 


\section{Bibliografía}

Batalla, R. J., Gomez, C. M., \& Kondolf, G. M. (2004). Reservoir-induced hydrological changes in the Ebro River basin (Northeastern Spain). Journal of Hydrology, 290(1-2), 117-136.

Batalla, R. J. \& Vericat D. (2009). Hydrological and sediment transport dynamics of flushing flows: implications for river management in large Mediterranean rivers. River Research and Applications, 25(3), 297-314.

Batalla, R. J., Vericat, D., Farguell, J., Úbeda, X., \& Garcia, C. (2020). Processos hidrològics i geomorfològics als rius: context i exemples per a interpretar la seva resposta a episodis d'alta magnitud com el Glòria. Treballs de la Societat Catalana de Geografia, 89, 55-87.

Batalla, R. J., Gibbins, C. N., Alcázar, J., Brasington, J., Buendia, C., Garcia, C., Llena, M., López, R., Palau, A., Rennie, C., Wheaton, J. M., \& Vericat, D. (2021). Hydropeaked rivers need attention. Environmental Research Letters, 16, 021001. doi.org/10.1088/1748-9326/abce26

Béjar, M., Vericart, D., Nogales, I., Gallart, F., \& Batalla, R. J. (2018). Efectos de las extracciones de áridos sobre el transporte de sedimentos en suspensión en ríos de montaña (alto río Cinca, Pirineo central). Cuadernos Investigación Geográfica, 44(2), 641-658.

Brasington, J., Vericat, D., \& Rychkov, I. (2012). Modelling river bed morphology, roughness and surface sedimentology using high resolution terrestrial laser scanning. Water Resources Research, 48, W11519, doi:10.1029/2012WR012223

Brown, A. V., Lyttle, M. M., \& Brown, K. B. (1998). Impacts of gravel mining on gravel bed streams. Transactions of the American Fisheries Society, 127, 979-994. http://doi.org/10.1577/1548-8659(199 8) 127<0979:IOGMOG>2.0.CO;2

Brownlie, W. R. \& Taylor, B. D. (1981). Sediment management for southern California mountains, coastal plains, and shoreline. Part C. Coastal sediment delivery by major rivers in southern California. Report 17-C, Environmental Quality Lab., California Institute of Technology, Pasadena.

Buendía, C., Bussi, G., Tuset, J., Vericat, D., Sabater, S., Palau, A., \& Batalla, R. J. (2016). Effects of afforestation on runoff and sediment load in an upland Mediterranean catchment. Science of the Total Environment, 540, 144-157.

Calvo, A., Gisbert, B., Palau, E., \& Romero, M. (1988). Un simulador de lluvia portátil de fácil construcción. En Sala, M. \& Gallart, F. (eds.). Métodos y técnicas para la medición de procesos geomorfológicos (pp. 6-15). Zaragoza: Sociedad Esp. Geomorfología, Monografía 1.

Carson, M. A. \& Kirkby, M. J. (1972). Hillslope form and process. Londres: Cambridge Univ. Press.

CEDEX (Centro de Estudios y Experimentación de obras públicas) (2011). Evaluación del impacto del cambio climático en los recursos hídricos en régimen natural. Ministerio de Medio Ambiente y Medio Rural y Marino.

Church, M. (2006). Bed material transport and the morphology of alluvial riverchannels. Annual Review Earth Planetary Sciences, 34, 325-354.

Conacher, A. J. \& Sala, M. (1998). Land degradation in mediterranean environments of the world: nature and extent, causes and solutions. Chichester: Wiley. 
Cotton, J. A., Wharton, G., Bass, J. A. B., Heppell, C. M., \& Wotton, R. S. (2006). The effects of seasonal changes to in-stream vegetation cover on patterns of flow and accumulation of sediment. Geomorphology, 77, 320-334.

Cowell, C. M. \& Dyer, J. M. (2002). Vegetation development in a modified riparian environament: Human imprint on an allegheny river wilderness. Annals of the Association of American Geographers, 92(2), 189-202.

De Castro, M., Martín-Vide. J., \& Alonso, S. (2005). El clima de España: pasado, presente y escenarios de clima para el siglo XXI. En Moreno-Rodríguez, J. M. (ed.). Evaluación preliminar de los impactos en España por efecto del cambio climático (pp. 1-65). Madrid: Ministerio de Medio Ambiente.

Dufour, S. \& Piégay, H. (2009). From the myth of a lost paradise to targeted river restoration: forget natural references and focus on human benefits. River Research and Applications, 25, 568-581.

Erskine, W. D. (1997). The real environmental costs of sand and soil mining on the Nepean River NSW. En Riley, S. J., Erskine, W. D., \& Shreshta, S. (eds.). Science and technology in the environmental management of the hawkesbury-nepean catchment (pp. 29-35). Geographical Society of New South Wales Conference Papers 14.

Erskine, W. D. \& Green, D. (2000). Geomorphic effects of extractive industries and their implications for river management: the case of the Hawkesbury-Nepean River, New South Wales. En Brizga, S. \& Finlayson, B. (eds.). River Management: The Australasian Experience (pp. 123-149). Chichester: John Wiley and Sons.

Gallart, F. \& Llorens, P. (2004). Observations on land cover changes and water resources in the headwaters of the Ebro catchment, Iberian Peninsula. Physics and Chemistry of the Earth, 29, 769-773.

Gibbins, C., Vericat, D., \& Batalla, R. J. (2007). When is stream invertebrate drift catastrophic? The role of hydraulics and sediment transport in initiating drift during flood events. Journal of Freshwater Biology, 52(12), 2369-2384.

Grove, A. T. \& Rackham, O. (2001). The nature of Mediterranean Europe: an ecological history. New Haven, CT / Londres: Yale University Press.

Inman, D. L. (1976). Man's impact on the California coastal zone. Summary Report to California Department of Navigation and Ocean Development. Sacramento.

IPCC (2013). Working Group I contribution to the IPCC 5th Assessment Report "Climate Change 2013: The Physical Science Basis". 12 ${ }^{\text {th }}$ Session of Working Group I and the $36^{\text {th }}$ Session of the IPCC, 26 de septiembre de 2013, Estocolmo (Suecia).

Kondolf, G. M. (1997). Hungry water: Effects of dams and gravel mining on river channels. Environmental Management, 21(4), 533-551.

Kondolf, G. M. \& Wilcock P. R. (1996). The flushing flow problem: defining and evaluating objectives. Water Resources Research, 32(8), 2589-2599.

Lane, E. W. (1955). The importance of fluvial geomorphology in hydraulic engineering. Proceedings of the American Society of Civil Engineers, 81, 1-17.

Leopold, L. B., Wolman, M. G., \& Miller J. P. (1964). Fluvial processes in geomorphology. San Francisco: W. H. Freeman and Co. 
Ligon, F. K., Dietrich, W. E., \& Trush, W. J. (1995). Downstream ecological effects of dams, a geomorphic perspective. Bioscience, 45(3), 183-192.

Marston, R. A., Bravard, J. P., \& Green, T. (2003). Impacts of reforestation and gravel mining on the Malnant River, Haute-Savoie, French Alps. Geomorphology, 55(1-4), 65-74.

Milly, P. C. D., Dunne, K. A., \& Vecchia, A. V. (2005). Global pattern of trends in streamflow and water availability in a changing climate. Nature, 438, 347-350.

Mimikou, M., Blatas, E., Varanaou, E., \& Pantazis K. (2000). Regional impacts of climate change on water resources quantity and quality indicators. Journal of Hydrology, 234, 95-109.

Morán-Tejeda, E., Ceballos-Barbancho, A., \& Llorente-Pinto, J. M. (2010). Hydrological response of Mediterranean headwaters to climate oscillations and land cover changes: the mountains of Duero River basin (Central Spain). Global and Planetary Change, 72, 39-49.

Piégay, H. \& Peiry, J. L. (1997). Long profile evolution of a mountain stream in relation to gravel load management: example of the middle Giffre River (French Alps). Environmental Management, 21(6), 909-919.

Poff, N. L. (1997). Landscape filters and species traits: towards mechanistic understanding and prediction in stream ecology. Journal of the North American Benthological Society, 16, 391-409.

Richards, K. (1982). Rivers. Form and process in alluvial channels. Londres: Methuen.

Rinaldi, M. \& Simon, A. (1998). Bed-level adjustments in the Arno River, Central Italy. Geomorphology, 22, 57-71.

Rovira, A., Batalla, R. J., \& Sala, M. (2005). Response of a river sediment budget after historical gravel mining (the lower Tordera, NE Spain). River Research and Applications, 21(7), 829-847.

Sabater, S. \& Barceló, D. (2012). Water scarcity in the Mediterranean: perspectives under global change. The Handbook of Environmental Chemistry.

Sala, M. \& Batalla, R. J. (1996). Teoría y métodos en geografía física. Madrid: Síntesis.

Sala, M. \& Gallart, F. (1988). Métodos y técnicas para la medición en el campo de procesos geomorfológicos. Sociedad Española de Geomorfología, Monografía 1.

Schälchli, U. (1992). The clogging of coarse gravel river beds by fine sediment. Hydrobiologia, 235, 189-197.

Scheidegger, A. E. (1961). Mathematical models of slope development. Bulletin of the Geological Society of America, 72, 37-49.

Slaymaker, O. (1980). Geomorphic field experiments. Inventory and prospect. Zeitschrift für Geomorphologie Supplement, 35, 183-194.

Vericat, D. \& Batalla, R. J. (2006). Sediment transport in a large impounded river: The lower Ebro, NE Iberian Peninsula. Geomorphology, 79, 72-92.

Vericat, D., Batalla, R. J., \& Gibbins, C. N. (2008). Sediment entrainment and exhaustion from patches of fine material in a gravel-bed river. Water Resources Research, 44, W11415, doi:10.1029/2008WR007028.

Williams, G. P. \& Wolman, M. G. (1984). Downstream effects of dams on alluvial rivers. US Geological Survey Professional Paper 1986. 
Yarnell, S. M., Petts, G. E., Schmidt, J. C., Whipple, A. A., Beller, E. E., Dahm, C. N., Goodwin, P., $\&$ Viers, J. H. (2015). Functional flows in modified riverscapes: hydrographs, habitats and opportunities. BioScience, 65(10), 963-972.

Cómo citar este artículo:

Batalla, R. J. (2021). Reflexión sobre dinámica morfosedimentaria. Implicaciones para la gestión fluvial en un contexto de cambio global. Cuadernos de Geografía, 107, 175-190.

https://doi.org/10.7203/CGUV.107.213372

\section{(9) $(\Theta \Theta \Theta$}

Este obra está bajo una licencia de Creative Commons Reconocimiento-NoComercial-SinObraDerivada 4.0 Internacional. 